OPEN ACCESS

Edited by:

Tzvi Dwolatzky,

Technion Israel Institute of

Technology, Israe

Reviewed by:

Vincent Hooper,

Xiamen University, China

Manoj Kumar Yadav,

Mahindra University, India

Anna Vittoria Mattioli;

University of Modena and Reggio

Emilia, Italy

*Correspondence:

Marios Kyriazis

drmarios@live.it

Specialty section

This article was submitted to

Geriatric Medicine

a section of the journal

Frontiers in Medicine

Received: 31 May 2021

Accepted: 12 July 2021

Published: 02 August 2021

Citation:

Kyriazis M, Mikellides $G$,

Pantelidakis $\mathrm{H}$, Polycarpou M and

Panayiotou B (2021) COVID-19

Isolation and Risk of Death in Cyprus

Elderly People. Front. Med. 8:717692.

doi: 10.3389/fmed.2021.717692

\section{COVID-19 Isolation and Risk of Death in Cyprus Elderly People}

\author{
Marios Kyriazis ${ }^{1 *}$, George Mikellides ${ }^{1}$, Heraklis Pantelidakis ${ }^{1}$, Marina Polycarpou ${ }^{2}$ and \\ Barnabas Panayiotou ${ }^{3}$
}

${ }^{1}$ National Gerontology Centre, Larnaca, Cyprus, ${ }^{2}$ Materia Group, Nicosia, Cyprus, ${ }^{3}$ Postgraduate School of Medicine, Keele University, Stoke-on-Trent, Staffordshire, United Kingdom

Social isolation is associated with a higher risk of morbidity and death in older people. The quarantine and social distancing measures due to Covid-19 imposed in most countries and particularly in Cyprus, aim to isolate individuals from direct contact with others. This has resulted in vulnerable older people being isolated at their places of residence for several months, while the recommendations for continuing lockdowns do not appear to be ending. The risk of death from causes other than those related to Covid-19 increases in such individuals and it is due to the effects of social isolation. We estimate that in the next years, there will be a significant increase in the death numbers of such older people in Cyprus. The health authorities must develop a program of support for these older individuals to include medical, social, physical, and psychological elements. Examples of such support are given here.

Keywords: COVID-19, social isolation, older people, death risk, Cyprus elderly, prevention of mortality

\section{INTRODUCTION}

As the spread of Severe Acute Respiratory Syndrome Coronavirus-2 (SARS-CoV-2) continues to affect Cyprus, the elderly population has remained, and it is likely to remain, in enforced isolation for a long time. This is in line with similar policies in other countries. For example, the recommendation in the United Kingdom has been that people aged 70 years and older should be isolated at home for several months (1). However, it is well-accepted that social isolation in such age groups is a significant risk factor for morbidity and mortality and is therefore a serious public health concern $(2,3)$. This isolation exacerbates a variety of problems affecting older people which includes cardiovascular, cognitive, autoimmune, psychological, and hormonal disturbances (4).

We already knew before the current pandemic that there is consistent evidence linking social isolation and loneliness to worse cardiovascular and mental health outcomes (5). Moreover, we knew in advance that living alone and social disengagement is associated with a $24-32 \%$ higher hazard for hospital admission for elderly people suffering from respiratory disease (6).

In a recent qualitative study in Cyprus (7), it was confirmed that social isolation during the Covid-19 era is directly correlated with loneliness, a negative emotion associated with a perceived gap between existing relationships and desired ones. Strongest risk factors for morbidity and death were loss of a loved one, inactive lifestyle even before Covid-19, and clinical depression.

We are already beginning to experience increases in the numbers of non-Covid-19 deaths, compared to previous years (8). Estimates indicate a $17.7 \%$ increase in the number of deaths in the USA during 2020, compared to those in 2019, with heart disease and cancer-related deaths being the top two causes (9). It is possible that social isolation, associated with missed hospital treatments 
and other reasons connected to the strict lockdown measures, contributed to such an increase in deaths (10).

\section{MORBIDITY AND MORTALITY}

Valtorta et al. (11), examined 5,397 people aged 50 years and above, for an average period of 5.4 years. They found that there was an association between loneliness and increased risk of cardiovascular disease. Their conclusion was that: "Loneliness is associated with an increased risk of developing coronary heart disease and stroke, independently of traditional cardiovascular disease risk factors. Our findings suggest that primary prevention strategies targeting loneliness could help to prevent cardiovascular disease." Another study of 479,054 people over 7 years (12) confirmed the above findings concluding that "Isolated and lonely persons are at increased risk of acute myocardial infarction (AMI) and stroke, and, among those with a history of AMI or stroke, increased risk of death."

In a study of 6,500 participants aged 52 years and above, selected from The English Longitudinal Study of Aging in the UK (13), it was reported that social isolation (a state of complete or near-complete lack of contact between an individual and society) was associated with elevated risk of mortality (Hazzard Ratio of 1.26). Other studies also found significant association between loneliness (a temporary and involuntary lack of contact with others) and increased mortality (14). The increased mortality is not only linked to cardiovascular conditions. For example, it has been shown that other problems such as impaired immunity, altered hypothalamic pituitary-adrenocortical activity, and a pro-inflammatory gene expression profile are linked to increased mortality in this patients (15).

Another issue that needs to be considered is that of increased psychological morbidity. Pshychotropic drug use is on the increase during the Covid-19 pandemic (16). It is known that isolated older people are more likely to overuse medication such as antidepressants, anxiolytics, sedatives, or hypnotics (17). Chronic overuse of hypnotics and anxiolytics can lead to confusional states, is linked to dementia (18), and is associated with falls that can lead to hip fractures, head injuries, and even death (19).

Evidence is now emerging that the Covid-19 pandemic has widespread negative effects on cognitive abilities and on the mental well-being of older people $(4,20,21)$. In addition, loneliness is significantly associated with a reduction in brain volume, particularly in areas associated with memory such as the left medial temporal lobe (22). Other problems include poor sleep patterns and a decline in executive function (15), all of which increase the risk of mortality in older people.

When thinking of socially isolated people, we tend to exclude people who live in institutional settings. However, people, and particularly people with dementia, living in institutional settings (residential and nursing homes) have suffered the strongest additional negative impact both due to the ban of external visitors, but also internal social distancing measures (guidelines to keep patients in their rooms instead of letting them socialize freely in common living areas, placing patients in single occupancy rooms when possible etc. (23)). Psychiatric symptoms, leading to overuse of psychiatric medications and negative side-effects such as tardive dyskinesia and akathisia, have also increased, accelerating the deterioration of these patients and leading to complications and death (24).

It is also notable that elderly people hospitalized with Covid19 have negative short and long -term effects that impair their functionality because of the associated fatigue, muscle weakness and sarcopenia that can exacerbate frailty, dependence and disability, making this vulnerable group even more vulnerable leading to a vicious cycle of isolation and dependence (25).

\section{THE CYPRUS EXPERIENCE}

Considering that cardiovascular disease is the leading cause of death in Cyprus, it is particularly important to realize that social isolation and loneliness may cause significant morbidity and mortality in these individuals. During 2018 there were 5,768 (all causes) deaths in Cyprus. Of these, 3,829 were people aged 75 years and older (26). The leading causes of death in Cyprus for the year 2017 were ischemic heart disease, stroke, Alzheimer's disease, lung cancer, diabetes, and chronic obstructive pulmonary disease (27). All of these conditions have enormous significance for older people as they are negatively affected by social isolation. Estimates of isolation-related mortality vary, with Hazzard Ratios (HR) of 1.26-3.7 (28). Even if we consider the lowest HR estimates we should expect substantial increases in the numbers of excess deaths of older Cypriot people, from causes other than Covid-19.

In addition to morbidity and mortality due to social isolation, there is an issue of extra deaths due to other effects of the lockdowns such as delayed diagnoses and lack of suitable followup for conditions other than Covid-19 (29). For instance, it is known that cancer risk in these situations is a real concern, particularly due to delayed diagnosis (30). We could apply the findings of this study to cancers in Cyprus. A total of 1,325 deaths occurred in Cyprus due to cancers in 2016 (31). Based on an approximate estimate of increased deaths by $10 \%$ (30), we could expect $\sim 130$ extra deaths in Cyprus per year due to delayed cancer diagnosis alone. As reduced contact with healthcare staff contributes to higher risk, efforts to address this issue are likely to lead to improved health outcomes. Therefore, there is an urgent need to implement preventative strategies for physical and mental health that may reduce death risk in older populations (21).

\section{MEASURES TO MITIGATE RISKS}

It is necessary to devise strategies for supporting older people at risk from social isolation and health deterioration. A series of interventions by the State in association with volunteers could provide such support for vulnerable older people. Initiatives that would be beneficial include the following:

- A reformed Government policy is urgently needed to allow relatives and carers of isolated older individuals to visit them (whether at home, in a care home or hospital) in order 
to attend to their basic psychological and physical health needs and help to prevent their deterioration. As Covid-19 PCR tests are widely available and have sensitivity of 7198\% (32), there is no reason why visitors with no Covid19 symptoms and a negative PCR cannot meet vulnerable individuals. In cases where extra reassurance is desired, a second negative PCR test is known to confer the highest accuracy (32). In addition, spreading population strategies in favor of vaccination against Covid-19 offers glimpses of hope to reassess isolation protocols.

- Primary care services can maintain regular contact with vulnerable individuals at high risk in the community, via telephone calls from healthcare professionals in order to ensure early identification of, and intervention for, medical and other needs. The calls should be initiated by the health professional and not by the patient (33). Likewise, provision of specialist psychological support can have beneficial outcomes (34). Furthermore, volunteers, friends, and relatives can enable meaningful and supportive telephone conversations on a regular basis (35).

- Promote physical activities, preferably a mixture of resistance, strength, and balance exercises as even light intensity exercise has positive health outcomes (36). For some individuals, technology such as use of the internet, social media sites, and media broadcasts can support these exercises programs (37). Some of these programs could be presented without charge by television stations within their sphere of corporate social responsibility.

- Consider "hidden" negative aspects of issues affecting older people, such as for example, the social stigma associated with Covid-19, negative perpetuating factors promoting the pandemic, and general fear (38).

- Smart ICT solutions, such as ReMember-Me (39). These may help prevent and detect cognitive decline, promote cognitive function and social inclusion among older adults. Smart solutions offer an innovative paradigm to improve cognition, emotional well-being, activity, sleep patterns and online socialization, promoting interactions in the context of cognitive fitness and individualized suggestions for a healthy brain.

\section{REFERENCES}

1. Centre for Evidence-Based Medicine. (2020). Available online at: https:// www.cebm.net/covid-19/maximising-mobility-in-the-older-people-whenisolated-with-covid-19/ (accessed April 11, 2021).

2. Cacioppo JT, Cacioppo S. Social relationships and health: the toxic effects of perceived social isolation. Soc Personal Psychol Compass. (2020) 8:5872. doi: $10.1111 / \mathrm{spc} 3.12087$

3. Webb L. COVID-19 lockdown: a perfect storm for older people's mental health. J Psychiatr Ment Health Nurs. (2021) 28:300. doi: 10.1111/jpm.12644

4. Santini Z, Jose P, York Cornwell E, Koyanagi A, Nielsen L, Hinrichsen C, et al. Social disconnectedness, perceived isolation, and symptoms of depression and anxiety among older Americans (NSHAP): a longitudinal mediation analysis. Lancet Public Health. (2020) 5:e62-70. doi: 10.1016/S2468-2667(19)30230-0

5. Leigh-Hunt N, Bagguley D, Bash K, Turner V, Turnbull S, Valtorta $\mathrm{N}$, et al. An overview of systematic reviews on the public health
- Digital technologies can be harnessed further, and opportunities include online social and entertainment activities, networking, religious services, and board games (40), as well as cognitive training exercises and video-games (41). However, there is an issue of inequality with regards access to such technologies, and not all able, older individuals can participate (42).

\section{CONCLUSION}

Social isolation due to Covid-19 restrictive measures, has been shown to have a negative impact on health outcomes, with increasing morbidity and mortality among older people. It is imperative to develop nationwide strategies to prevent adverse outcomes, other than those related to Covid19 , in socially isolated people. In our attempt to prevent Covid-19 related deaths, we should not cause additional morbidity and deaths resulting from the isolation measures we have instigated. A wide range of interventions can be implemented that can promote social engagement and interactions, improve physical functions and psychological well-being, maintain optimal health status, and prevent deterioration. Although the examples of such interventions are best suited to the older people in Cyprus, the basic principles can be applicable to older people living in any country.

\section{DATA AVAILABILITY STATEMENT}

The original contributions presented in the study are included in the article/supplementary material, further inquiries can be directed to the corresponding author/s.

\section{AUTHOR CONTRIBUTIONS}

MK conceptualized and wrote the manuscript. GM, HP, and MP edited, revised, and added to the manuscript. BP edited and revised the manuscript and enhanced the concept. All approved the final version of the manuscript. consequences of social isolation and loneliness. Public Health. (2017) 152:15771. doi: 10.1016/j.puhe.2017.07.035

6. Bu F, Philip K, Fancourt D. Social isolation and loneliness as risk factors for hospital admissions for respiratory disease among older adults. Thorax. (2020) 75:597-9. doi: 10.1136/thoraxjnl-2019-214445

7. Hadjicosta A. Digi-Aging, Overcoming Loneliness, Erasmus 2020-1-AT01KA202-078084. (2021). Available online at: https://ec.europa.eu/programmes/ erasmus-plus/projects/eplus-project-details/\#project/2020-1-AT01-KA202078084 (preliminary data) (accessed July 1, 2021).

8. Woolf SH, Chapman DA, Sabo RT, Weinberger DM, Hill L, Taylor DDH. Excess deaths from COVID-19 and OTHER CAUSES, MARCH-July 2020. JAMA. (2020) 324:1562-4. doi: 10.1001/jama.2020.19545

9. Ahmad FB, Anderson RN. The leading causes of death in the US for 2020. JAMA. (2021) 325:1829-30. doi: 10.1001/jama.2021.5469

10. Young MN, Iribarne A, Malenka D. COVID-19 and cardiovascular health. J Am Coll Cardiol. (2021) 77:170-2. doi: 10.1016/j.jacc.2020.11.027 
11. Valtorta NK, Kanaan M, Gilbody S, Hanratty B. Loneliness, social isolation and risk of cardiovascular disease in the english longitudinal study of ageing. Eur J Prev Cardiol. (2018) 25:1387-96. doi: 10.1177/2047487318792696

12. Hakulinen C, Pulkki-Råback L, Virtanen V, Jokela M, Kivimäki M, Elovainio $\mathrm{M}$, et al. Social isolation and loneliness as risk factors for myocardial infarction, stroke and mortality: UK biobank cohort study of 479054 men and women. Heart. (2018) 104:1536-42. doi: 10.1136/heartjnl-2017-312663

13. Steptoe A, Shankar A, Demakakos P, Wardle J. Social isolation, loneliness, and all-cause mortality in older men and women. Proc Natl Acad Sci USA. (2013) 110:5797-801. doi: 10.1073/pnas.1219686110

14. Tabue Teguo M, Simo-Tabue N, Stoykova R, Meillon C, Cogne M, Amiéva $\mathrm{H}$, et al. Feelings of loneliness and living alone as predictors of mortality in the elderly: the PAQUID study. Psychosom Med. (2016) 78:9049. doi: 10.1097/PSY.0000000000000386

15. Hawkley LC, Capitanio JP. Perceived social isolation, evolutionary fitness and health outcomes: a lifespan approach. Philos Trans R Soc Lond B Biol Sci. (2015) 370:20140114. doi: 10.1098/rstb.2014.0114

16. Rauf B, Sheikh H, Majid H, Roy A, Pathania R. COVID-19-related prescribing challenge in intellectual disability. Br J Psych Open. (2021) 7:e66. doi: 10.1192/bjo.2021.26

17. Canham SL. What's loneliness got to do with it? Older women who use benzodiazepines. Austral J Ageing. (2015) 34:E7-12. doi: 10.1111/ajag.12133

18. Billioti de Gage S, Moride Y, Ducruet T, Kurth T, Verdoux H, Tournier M, et al. Benzodiazepine use and risk of Alzheimer's disease: case-control study. BMJ. (2014) 349:g5205. doi: 10.1136/bmj.g5205

19. Kripke DF. Hypnotic drug risks of mortality, infection, depression, and cancer: but lack of benefit. F1000Res. (2016) 5:918. doi: 10.12688/f1000research.8729.1

20. Manca R, De Marco M, Venneri A. The impact of COVID-19 infection and enforced prolonged social isolation on neuropsychiatric symptoms in older adults with and without dementia: a review. Front Psychiatry. (2020) 11:1086. doi: 10.3389/fpsyt.2020.585540

21. Armitage R, Nellums LB. COVID-19 and the consequences of isolating the elderly. Lancet. (2020) 5:E256. doi: 10.1016/S2468-2667(20)30061-X

22. Düzel S, Drewelies J, Gerstorf D, Demuth I, Steinhagen-Thiessen E, Lindenberger U, et al. Structural brain correlates of loneliness among older adults. Sci Rep. (2019) 9:13569. doi: 10.1038/s41598-019-49888-2

23. Comas-Herrera A, Zalakaín J, Lemmon E, Henderson D, Litwin C, Hsu AT, et al. Mortality Associated With COVID-19 in Care Homes: International Evidence 2020. London: Article in LTCcovid.org, International Long-Term Care Policy Network, CPEC-LSE (2021).

24. European Centre for Disease Prevention and Control (ECDC). COVID19 Surveillance Report. Available online at: https://www.ecdc.europa.eu/en/ covid- 19 (accessed May 15, 2021).

25. Sagarra-Romero L, Viñas-Barros A. COVID-19: short and long-term effects of hospitalization on muscular weakness in the elderly. Int J Environ Res Public Health. (2020) 17:8715. doi: 10.3390/ijerph17238715

26. Ministry of Finance Statistical Service, Cyprus. Age-Specific Mortality Rates. (2018). Available online at: https://www.mof.gov.cy/mof/cystat/statistics. nsf/populationcondition_21main_en/populationcondition_21main_en? OpenForm\&sub=1\&sel=4 (accessed April 11, 2021)

27. The Institute for Health Metrics and Evaluation, Cyprus 2017. Available online at: http://www.healthdata.org/cyprus (accessed May 12, 2021).

28. Manemann S, Chamberlain AM, Roger LV, Griffin JM, Boyd CM, Cudjoe TKM, et al. Perceived social isolation and outcomes in patients with heart failure. J Am Heart Assoc. (2018) 7:e008069. doi: 10.1161/JAHA.117.008069

29. Hanna TP, King WD, Thibodeau S, Jalink M, Paulin GA, Harvey-Jones E, et al. Mortality due to cancer treatment delay: systematic review and meta-analysis. BMJ. (2020) 371:m4087. doi: 10.1136/bmj.m4087

30. Maringe C, Spicer J, Morris M, Purushotham A, Nolte E, Sullivan R, et al. The impact of the COVID-19 pandemic on cancer deaths due to delays in diagnosis in England, UK: a national, population-based, modelling study. Lancet Oncol. (2020) 21:1023-34. doi: 10.1016/S1470-2045(20)30388-0

31. Ministry of Health, Cyprus, Statistics/cancer 2016. Available online at: https://www.moh.gov.cy/Moh/MOH.nsf/All/ 82B40CE2FEE19D7AC22579C6002CBFAD?OpenDocument (accessed May 2, 2021).

32. Watson J. Interpreting a covid-19 test result. BMJ. (2020) 369:m1808. doi: 10.1136/bmj.m1808

33. Abrashkin KA, Zhang J, Poku A. Acute, post-acute, and primary care utilization in a home-based primary care program during COVID-19. Gerontologist. (2021) 61:78-85. doi: 10.1093/geront/gn aa158

34. WHO. Mental Health and Psychosocial Considerations During the COVID19 outbreak. (2020). Available online at: https://www.who.int/docs/defaultsource/coronaviruse/mental-health-considerations.pdf (accessed April 12, 2021).

35. Newman M, Zainal N. The value of maintaining social connections for mental health in older people. Lancet Public Health. (2020) 5:e12e3. doi: 10.1016/S2468-2667(19)30253-1

36. Hartmann-Boyce J, Davies N, Frost R, Park S. The Centre for Evidence-Based Medicine. Maximising Mobility in Older People When Isolated With COVID 19. (2020). Available online at: https://www.cebm.net/covid-19/maximisingmobility-in-the-older-people-when-isolated-with-covid-19/ (accessed May 3, 2021).

37. Hajek A, König Hand-Helmut. Social isolation and loneliness of older adults in times of the COVID-19 pandemic: can use of online social media sites and video chats assist in mitigating social isolation and loneliness? Gerontology. (2021) 67:121-4. doi: 10.1159/000512793

38. WHO. A Guide to Preventing and Addressing Social Stigma Associated With COVID-19. Available online at: https://www.who.int/publications/m/item/aguide-to-preventing-and-addressing-social-stigma-associated-with-covid19 (accessed April 12, 2021).

39. ReMember-Me (EU funded project AAL-2019-6-188-CP). (2020). Available online at: http://www.aal-europe.eu/projects/remember-me/, and from: https://www.rememberme-aal.eu/ (accessed May 5, 2021).

40. Brooke J, Jackson D. Older people and COVID-19: Isolation, risk and ageism. J Clin Nurs. (2020) 29:2044-6. doi: 10.1111/jocn.15274

41. Kyriazis M, Kiourti E. Video games and other online activities may improve health in ageing. Front Med. (2018) 5:8. doi: 10.3389/fmed.2018.00008

42. Käll A, Jägholm S, Hesse H, Andersson F, Mathaldi A, Norkvist BT, et al. Internet-based cognitive behavior therapy for loneliness: a pilot randomized controlled trial. Behav Ther. (2020) 51:54-68. doi: 10.1016/j.beth.2019.05.001

Conflict of Interest: The authors declare that the research was conducted in the absence of any commercial or financial relationships that could be construed as a potential conflict of interest.

Publisher's Note: All claims expressed in this article are solely those of the authors and do not necessarily represent those of their affiliated organizations, or those of the publisher, the editors and the reviewers. Any product that may be evaluated in this article, or claim that may be made by its manufacturer, is not guaranteed or endorsed by the publisher.

Copyright (c) 2021 Kyriazis, Mikellides, Pantelidakis, Polycarpou and Panayiotou. This is an open-access article distributed under the terms of the Creative Commons Attribution License (CC BY). The use, distribution or reproduction in other forums is permitted, provided the original author(s) and the copyright owner(s) are credited and that the original publication in this journal is cited, in accordance with accepted academic practice. No use, distribution or reproduction is permitted which does not comply with these terms. 